\title{
Taeniasis-cysticercosis in Southern Ecuador: assessment of infection status using multiple laboratory diagnostic tools
}

\author{
R Rodriguez-Hidalgo, W Benitez-Ortiz, N Praet*, LR Saa**, J Vercruysse***, \\ J Brandt*, P Dorny*/+
}

\begin{abstract}
Centro Internacional de Zoonosis, Universidad Central del Ecuador, Quito, Ecuador *Department of Animal Health, Prince Leopold Institute of Tropical Medicine Nationalestraat 155, B-2000 Antwerp, Belgium **Laboratorio de Servicios Agropecuarios, Escuela de Ciencias Agropecuarias, Universidad Técnica Particular de Loja, San Cayetano Alto, Loja, Ecuador ***Department of Virology, Parasitology and Immunology, Faculty of Medicine Veterinary, Gent University, Merelbeke, Belgium

Taenia solium-taeniasis and cysticercosis were studied in the human and porcine populations of a rural community in the Southern Ecuadorian Andes. From the 1059 inhabitants, 800 serum samples and 958 stool samples could be collected. In addition, 646 from the estimated 1148 pigs were tongue inspected. Circulating antigen was detected by enzyme linked immunosorbent assay (Ag-ELISA) in 2.25\% of the human population, whereas intestinal taeniasis was detected in $1.46 \%$ by the formalin-ether technique. Following treatment and recovery of tapeworm fragments these were all identified as T. solium. Porcine cysticercosis was diagnosed in $3.56 \%$ of the pigs by tongue inspection.

In addition, enzyme linked immunoelectrotransfer blot (EITB) was performed on a subset group of 100 humans to confirm the results of the Ag-ELISA. One hundred serum samples from pigs were also analysed by EITB. It appeared that 43 and $74 \%$ of humans and pigs had antibodies against $\mathrm{T}$. solium cysticerci, respectively. It is concluded that contrary to the high exposure of the human population to T. solium that is suggested by EITB, the number of active cysticercosis cases, diagnosed by Ag-ELISA, was low, which may indicate endemic stability. The further use of complementary diagnostic methods for a better understanding of the epidemiology of $\mathrm{T}$. solium is suggested.
\end{abstract}

Key words: Taenia solium - taeniasis - cysticercosis - enzyme linked immunosorbent assay - enzyme-linked immunoelectrotransfer blot - polymerase chain reaction - restricted fragment length polymerase - Ecuador

Taenia solium-taeniasis and cysticercosis are diseases present in developing countries, which are associated with social and environmental conditions such as lack of latrines, poor hygiene, and free roaming pigs with access to human faeces (WHO 2002). These conditions are common in the Andes, including Ecuador, where T. solium infections are endemic. Rodriguez-Hidalgo et al. (2003) carried out a survey in Imbabura, in the North of Ecuador where the temperate climatic conditions allow keeping pigs in farming conditions characterised by tethering or corralling. In this region, the prevalence of taeniasis and cysticercosis in humans was 1.05 and $5.11 \%$, respectively. The prevalence of porcine cysticercosis was $9.01 \%$.

While Rodriguez-Hidalgo et al. (2003) used antigen detection to determine cysticercosis prevalence, most studies in Latin America used antibody detection for immunodiagnosis (e.g. Gonzalez et al. 1990, Diaz et al. 1992, Garcia Noval et al. 1996, Sanchez et al. 1997, Goodman et al. 1999, Garcia et al. 2003, Cruz-Licea et al. 2003, PrestesCarneiro et al. 2006). Both methods attempt to measure the proportion of infected individuals, however, antigen detection is restricted to the detection of viable cysticerci, while the finding of antibodies can indicate the presence

${ }^{+}$Corresponding author: pdorny@itg.be

Received 18 May 2006

Accepted 6 September 2006 of both viable and degenerated cysticerci or past infection (Dorny et al. 2004a). Moreover, Garcia et al. (2001) demonstrated that in cysticercosis-endemic areas of Peru and Colombia approximately $40 \%$ of people, seropositive by enzyme-linked immunoelectrotransfer blot (EITB) had become seronegative when resampled after 1 to 3 years. This indicated that transient antibodies might occur following exposure to $T$. solium eggs.

The aim of this study was to evaluate the cysticercosis/taeniasis situation in an endemic community in the southern Andes, where conditions are prone to transmission of T. solium, i.e. extensive pig husbandry and few or no latrines. We aimed to sample the entire human and pig populations in a defined community. Serum samples of man were screened with an antigen detecting test, and stool samples by coprological examination. Pigs were examined by tongue palpation. In addition, antibody tests on serum samples were performed on sub-populations of man and pigs.

\section{MATERIALS AND METHODS}

Study area and population - The study was done in Limones $\left(80^{\circ} 21^{\prime} \mathrm{W}, 4^{\circ} 23^{\prime} \mathrm{S}\right)$, a rural community (about 35 $\mathrm{km}^{2}$ ) in the southern Andean province of Loja, bordering Peru. The region has two well-defined climatic seasons, i.e. a rainy season between December and May and a dry season during the rest of the year (average annual rainfall $472 \mathrm{~mm}$ and average annual temperature $26^{\circ} \mathrm{C}$ ). Pig rearing is marginal in this arid region: the animals are left free roaming in search of feed during the day time and confined at night and usually kept only for local consump- 
tion (Benitez-Ortiz 2001). According to preliminary censuses in January 2003, Limones is composed of 17 neighbourhoods with 222 families. In total, 1059 inhabitants and 1148 pigs were recorded.

Experimental design - The survey was carried out during the dry season (July-August) of 2003. The local authorities and community leaders were contacted and informed about the project. Each individual household was visited and all persons between 2 and 80 years were invited to participate in the survey. Vials for collecting stool samples were distributed to be collected the next day together with a blood sample taken at the local health centres.

Taenia carriers who were identified following examination of the stool samples were offered cestocidal treatment, i.e. a single oral dose of praziquantel at $10 \mathrm{mg} / \mathrm{kg}$ b.w. and a tablet of laxative "Dulcolax" (Boehringer Ingelheim $\mathrm{GmbH}$, Germany). Following treatment, tapeworms or their fragments were collected from the stools and stored in ethanol $70 \%$ for species differentiation. People who were negative for Taenia spp. eggs but appeared to be infected with gastrointestinal nematodes were offered a single dose of albendazole $(400 \mathrm{mg})$.

Pig owners were requested to keep their animals penned. From the estimated 1148 total number of pigs in the community, 646 were submitted to the visual inspection of the ventral face of the tongue (Gonzalez et al. 1990). Pigs positive to tongue inspection were treated immediately with a single oral dose of oxfendazole $(30 \mathrm{mg} / \mathrm{kg}$ b.w) (Gonzalez et al. 1996).

A computer-generated random sample of 100 persons was selected among the participants. The serum samples from these persons were analysed by EITB (Tsang et al. 1989) for the detection of circulating antibodies against $T$. solium cysticerci.

One hundred pig serum samples were collected from the external jugular vein as described by Framstad et al. (2004) and examined by EITB, including 23 tongue-positive pigs and 77 randomly selected tongue-negative pigs.

The protocol of this survey was approved by the Ethical Committee of the Central University of Ecuador.

Diagnostic methods - Blood samples were centrifuged and the sera were transferred at $4^{\circ} \mathrm{C}$ to Quito-Ecuador where they were frozen at $-20^{\circ} \mathrm{C}$ until analysis. Tongue inspection was performed in pigs, visually and by palpating the ventral lingual surface as described by Gonzalez et al. (1990). Faecal samples were analyzed by the formalin-ether method (Ritchie 1948). Tapeworms collected from human stools following praziquantel treatment were differentiated by morphological characteristics, glucose phosphate isomerase (GPI) patterns and polymerase chain reaction-restricted fragment length polymerase (PCRRFLP) as described by Rodriguez Hidalgo et al. (2002).

Sera from humans were tested in an Ag-ELISA (Brandt et al. 1992) with the modifications, including pre-treatment of the sera by trichloroacetic acid and the interpretation of the results, as described by Dorny et al. (2000) and Erhart et al. (2002). Because a preliminary experiment, consisting in the dissection of the entire carcass of five pigs that were found positive in the Ag-ELISA, revealed that two pigs were infected with metacestodes of $T$. solium, another two with $T$. hydatigena and one pig with both parasites, it was decided not to use this test for serodiagnosis. T. hydatigena infections in pigs are known to crossreact in the Ag-ELISA (Dorny et al. 2004b).

Human and porcine sera were tested, at the Cayetano Heredia University in Lima-Peru, by EITB (Tsang et al. 1989), for antibodies against seven T. solium glycoprotein antigens.

\section{RESULTS}

Human population - From the 1059 inhabitants, 800 serum samples and 958 stool samples could be collected. A total of 18 sera [2.25\% (CI 95\%: 1.34-3.53)] were positive for $T$. solium metacestode circulating antigens (age range from 5 to 78 years). From the 958 faecal samples $14[1.46 \%$ (CI 95\%: 0.80-2.44)] were positive for Taenia spp. eggs by coprological examination (age range from 12 to 73 years). All except two positives were from separate households. Following treatment of these carriers, 12 worms were collected. All of these worms were identified as T. solium. Among these tapeworm carriers four individuals were positive by Ag-ELISA. One of these individuals who was positive in both Ag-ELISA and EITB developed clinical symptoms, i.e. severe headache and epileptic seizures, shortly after treatment for taeniasis. He was referred to a hospital in Loja city where neurocysticercosis (NCC) was confirmed by CT-scan and where he was treated.

From the subset of human serum samples collected for further analysis, six samples were positive by Ag-ELISA while 40 samples were positive by EITB. In the EITB, three samples recognised all seven glycoproteins (GP), while two samples showed 5 bands, two samples 4 bands, 16 samples three bands, 7 samples two bands and 10 samples only one band. The proteins most frequently recognised were the GP50 (85\%), GP39-42 complex (88\%) and GP 24 (90.5\%); the lower molecular weight proteins (GP21 (10\%), GP18 (10\%), GP14 (7.5\%) and GP13 (17.5\%)) were less recognised. From the six samples positive by Ag-ELISA, only two were positive by EITB, showing one (GP50) and three bands (GP50, GP39-42, GP24), respectively.

Pigs - From the 646 pigs examined, 23 [3.56\% (CI 95\%: 2.27-5.29)] had between one and 20 cysts in the sublingual surface.

In the subset of 100 pigs 74 serum samples were positive in EITB, including the 23 pigs with cysticerci at tongue inspection and 51 tongue negative pigs. Like for the human samples the proteins most frequently recognised in the EITB were the GP50 (97\%), GP39-42 complex (75\%), and GP 24 (72.5\%); the lower molecular weight proteins [GP21 (5.5\%), GP18 (7\%), GP14 $(1.5 \%)$ and GP13 (12\%)] were less recognised. None of the samples recognised all seven GP, three samples reacted with six bands, one with five bands, seven with four bands, 46 with three bands, five with two bands and 12 with one band.

\section{DISCUSSION}

This study demonstrated the presence of T. solium in humans and pigs in a rural community in the southern Andes of Ecuador. More than $80 \%$ of the human popula- 
tion participated in the study and more than $56 \%$ of the pigs could be inspected by tongue examination. Therefore, we consider this sample to be representative for the human and pig populations in the surveyed community.

Taeniasis was detected in $1.46 \%$ of the human population by microscopic examination; which is slightly higher than the results from similar studies in the North of Ecuador (Rodriguez-Hidalgo et al. 2003). However, all Taenia in this study were identified as T. solium whereas in the North only eight out of 29 worms collected were T. solium, the other 21 worms being identified as T. saginata.

With this higher percentage of T. solium tapeworms in the population also a higher percentage of human and porcine cysticercosis cases would be expected. Nevertheless, the percentage of Ag-ELISA positives in humans was lower than in the study in the North. In contrast, the percentage of tongue-positive pigs in the South was higher $(3.56 \%)$ than the number of carcass inspection-positive pigs detected in the North $(0.52 \%)$. However, tongue and meat inspection have an equally low sensitivity (Dorny et al. 2004b), therefore, these figures probably seriously underestimate the real prevalence of porcine cysticercosis in both regions.

EITB performed on a sub-set of 100 randomly selected individuals indicated a very high proportion of the human population carrying antibodies against $T$. solium: among these 100 individuals only six had circulating antigens and more than $40 \%$ had circulating antibodies. The proportion of antibody positive pigs among the randomly selected tongue negatives was also very high $[66 \%$ (CI 95\%: 54-77)].

While Ag-ELISA measures active cysticercosis, detection of circulating antibodies indicates exposure and thus not merely active infection (Dorny et al. 2004a). Thus, a positive antibody test would indicate infection, active or past, or a transient antibody response (Garcia et al. 2001, Gonzalez et al. 2006). The high proportion of antibody positives in humans and in pigs would reflect high exposure rate in the study area. Indeed, risk factors for taeniasis-cysticercosis such as open-air defecation, free roaming of pigs, and the eating of undercooked pork are very prominent in Loja province. In this area pigs have been seen to cover wide distances for finding feed and water, which increases the risk of infection of pigs.

The data shown in this study suggest that frequency of contact with oncospheres may be an important factor in the development of endemic stability (Gonzalez et al. 2006). This has been described in other parasitoses such as malaria and lymphatic filariasis (Coluzzi 1999, Melrose 2002). In the context of human cysticercosis "endemic normal" (Melrose 2002) would thus refer to people who, despite regular exposure to oncospheres and subsequent development of an antibody response, have only temporarily or never developed viable cysticerci in the CNS, hence do not show clinical evidence of disease. The clinical prevalence of $\mathrm{NC}$ in this region merits further studies; however, preliminary results indicate low involvement of $\mathrm{NC}$ in epilepsy cases.

More data are needed to support the hypothesis of endemic stability in cysticercosis, yet, these observations point to the importance to differentiate between persons with active cysticercosis and those having been exposed to oncospheres, i.e. the simultaneous use of antigen and antibody detection, ideally supported by neuro-imaging.

As a final conclusion, high exposure of humans and pigs to oncospheres of T. solium was demonstrated in a region where the harsh climatic conditions are a major constraint for agriculture, which directly affects pig husbandry. In spite of the limited number of samples that could be analyzed by EITB, it was demonstrated that a high percentage of humans and pigs have antibodies against the parasite. Most of the infected individuals, however, did not show any evidence of NC. In addition, our findings point out the difference of interpretation of test results based on direct and indirect immunodiagnostic methods and suggest the use of multiple diagnostic methods to determine the true prevalence of the disease. This multi-testing based diagnosis will allow a better understanding of the epidemiology of the taeniasis-cysticercosis complex and may also support individual diagnosis.

\section{ACKNOWLEDGEMENTS}

To A Gonzalez and H Garcia who kindly performed EITB test in Peru. To the technical staff of the Centro Internacional de Zoonosis in Quito and the Department of Animal Health of ITM, Antwerp, in particular Margoth Barrionuevo, Maritza Celi, Anke van Hul, and Bjorn Victor, and to the patients for their willingness to participate in this study. This research was part of a joint Ecuadorian-Belgian project, funded by Framework agreement IMT/CIZ 96122 of the Belgian General Directorate for Development Co-operation (DGDC) by the Central University of Ecuador and the Institute of Tropical Medicine in Belgium. The experiments comply with the current laws of Belgium and Ecuador where the experiments were performed.

\section{REFERENCES}

Benitez-Ortiz W 2001. Los cerdos criollos ecuatorianos. In Los Cerdos Locales en los Sistemas Tradicionales de Producción, FAO, Roma, p. 37-70.

Brandt JRA, Geerts S, De Deken R, Kumar V, Ceulemans F, Brijs L, Falla N 1992. A monoclonal antibody-based Elisa for the detection of circulating excretory-secretory antigens in Taenia saginata cysticercosis. Int J Parasitol 22: 471-477.

Coluzzi M 1999. The clay feet of the malaria giant and its African roots: hypotheses and inferences about origin, spread and control of Plasmodium falciparum. Paras-sitologia 41: 277-283.

Cruz-Licea V, Plancarte-Crespo A, Moran I, Valencia S, Rodriguez G, Vega L 2003. Teniosis y cisticercosis en comerciantes de alimentos enmercados de una área de la ciudad de México. Parasitol Latinoamericana 58: 41-48.

Diaz F, Garcia HH, Gilman RH, Gonzalez AE, Castro M, Tsang VCW, Pilcher JB, Vasquez LE, Lescano M, Carcamo C, Madico G, Miranda E 1992. Epidemiology of taeniasis and cysticercosis in a Peruvian village. Am J Epidemiol 135: 875882.

Dorny P, Brandt J, Geerts S 2004a. Immunodiagnostic approaches for detecting Taenia solium. Trends Parasitol 20: 259-260.

Dorny P, Phiri IK, Vercruysse J, Gabriel S, Willingham III AL, Brandt J, Victor B, Speybroeck N, Berkvens D 2004b. A Bayesian approach for estimating values for prevalence and 
diagnostic test characteristics of porcine cysticercosis. Int $J$ Parasitol 34/5: 569-576.

Dorny P, Vercammen F, Brandt J, Vansteenkiste W, Berkvens D, Geerts S 2000. Sero-epidemiological study of Taenia saginata cysticercosis in Belgian cattle. Vet Parasitol 88 : 43-49.

Erhart A, Dorny P, Van De N, Vien HV, Thach DC, Toan ND, Cong L, Geerts S, Speybroeck N, Berkvens D, Brandt J 2002. Taenia solium cysticercosis in a village in northern Viet Nam: seroprevalence study using an ELISA for detecting circulating antigen. Trans R Soc Trop Med Hyg 96: 270272.

Framstad T, Sjaastad O, Aass RA 2004. Bleeding and intravenous techniques in pigs. http://oslovet. veths. no/teaching/ pig/pigbleed/.

Garcia HH, Gilman RH, Gonzalez AE, Verastegui M, Rodriguez S, Gavidia C, Tsang VCW, Falcon N, Lescano AG, Moulton LH, Bernal T, Tovar M, Cysticercosis Working Group in Peru 2003. Hyperendemic human and porcine Taenia solium infection in Peru. Am J Trop Med Hyg 68: 268-275.

Garcia HH, Gonzalez AE, Gilman RH, Palacios LG, Jimenez I, Rodriguez S, Verastegui M, Wilkins P, Tsang VCW, The Cysticercosis Working Group in Peru 2001. Transient antibody response in Taenia solium infection in field conditions - A major contributor to high seroprevalence. Am J Trop Med Hyg 65: 31-32.

Garcia-Noval J, Allan JC, Fletes C, Moreno E, Demata F, Torresalvarez R, Dealfaro HS, Yurrita P, Higueros Morales H, Mencos F, Craig PS 1996. Epidemiology of Taenia solium taeniasis and cysticercosis in two rural Guatemalan communities. Am J Trop Med Hyg 55: 282-289.

Gonzalez AE, Cama V, Gilman RH, Tsang VCW, Pilcher JB, Chavera A, Castro M, Montenegro T, Verastegui M, Miranda E, Bazalar H 1990. Prevalence and comparison of serologic assays, necropsy, and tongue examination for the diagnosis of porcine cysticercosis in Peru. Am J Trop Med Hyg 43: 194-199.

GonzalezAE, Garcia HH, Gilman RH, Gavidia CH, Tsang VCW, Bernal T, Falcon N, Romero M, Lopezurbina MT 1996. Effective, single-dose treatment of porcine cysticercosis with oxfendazole. Am J Trop Med Hyg 54: 391-394.
Gonzalez AE, Ramos D, Gavidia CM, Guezala C, Romero M 2006. A porcine cysticercosis survey in an endemic region: new evidence in disease aggregation. 11th International Congress of Parasitology, 7-11 August 25, Glasgow, Scotland, poster 1898 .

Goodman KA, Ballagh SA, Carpio A 1999. Case-control study of seropositivity for cysticercosis in Cuenca, Ecuador. Am J Trop Med Hyg 60: 70-74.

Melrose WD 2002. Lymphatic filariasis: new insights into an old disease. Int J Parasitol 32: 947-960.

Prestes-Carneiro LE, Freitas Sde B, Zago SC, Miguel NA, Primo OB, Iha AH, Espindola NM, Vaz AJ 2006. Taeniosis-cysticercosis complex in individuals of a peasants' settlement (Teodoro Sampaio, Pontal of Paranapanema, SP, Brazil). Mem Inst Oswaldo Cruz 101: 15-20.

Ritchie LS 1948. An ether sedimentation technique for routine stool examinations. Bull US Army Med Depart 8: 326.

Rodriguez-Hidalgo R, Benitez-Ortiz W, Dorny P, Geerts S, Geysen D, Ron-Ronan J, Proano-Perez F, Chavez-Larrea MA, Barrionuevo-Samaniego M, Celi-Erazo M, VizcainoOrdonez L, Brandt J 2003. Taeniosis-cysticercosis in man and animals in the Sierra of Northern Ecuador. Vet Parasitol 118: 51-60.

Rodriguez-Hidalgo R, Geysen D, Benitez-Ortiz W, Geerts S, Brandt J 2002. Comparison of conventional techniques to differentiate between Taenia solium and Taenia saginata and an improved polymerase chain reaction-restriction fragment length polymorphism assay using a mitochondrial $12 \mathrm{~S}$ rDNA fragment. J Parasitol 88: 1007-1011.

Sanchez, AL, Gomez O, Allebeck P, Cosenza H, Ljungstrom I 1997. Epidemiological study of Taenia solium infections in a rural village in Honduras. Ann Trop Med Parasitol 91: 163-171.

Tsang VCW, Brand JA, Boyer AE 1989. An enzyme-linked immunoelectrotransfer blot assay and glycoprotein antigens for diagnosing human cysticercosis (Taenia solium). J Infect Dis 159: 50-59.

WHO 2002. Control of neurocysticercosis. In Fifty-fifth world health assembly, Provisional agenda item 13.18. A55/23. 